\title{
Odour Identification in Frontotemporal Lobar Degeneration
}

\author{
Lorena Rami $^{1,2}$, Clement T. Loy ${ }^{1,3}$, Julia Hailstone ${ }^{1}$, and Jason D. Warren ${ }^{1}$ \\ 1 Dementia Research Centre, Institute of Neurology, London, United Kingdom \\ 2 Memory-Alzheimer Unit, Department of Neurology, Hospital Clinic i Universitari Barcelona, Spain \\ 3 Neurobiology Program, Garvan Institute of Medical Research, 384 Victoria St, Darlinghurst, Sydney, \\ Australia
}

\begin{abstract}
Little information is available concerning olfactory processing in frontotemporal lobar degeneration (FTLD). We undertook a case-control study of olfactory processing in three male patients fulfilling clinical criteria for FTLD. Odour identification (semantic analysis) and odour discrimination (perceptual analysis) were investigated using tests adapted from the University of Pennsylvania Smell Identification Test. General neuropsychometry and structural volumetric brain magnetic resonance imaging (MRI) were also performed. The three patients with FTLD exhibited a disorder of olfactory processing with the characteristics of a predominantly semantic (odour identification) deficit. This olfactory deficit was more prominent in patients with greater involvement of the temporal lobes on MRI. Central deficits of odour identification may be more common in FTLD than previously recognised, and these deficits may assist in clinical characterisation.
\end{abstract}

\section{Keywords}

olfaction; odour; frontotemporal lobar degeneration; frontotemporal dementia

\section{Introduction}

Frontotemporal lobar degeneration (FTLD) is a group of neurodegenerative diseases characterised by progressive focal frontal and temporal lobe atrophy which together constitute a common cause of dementia in younger age groups [1]. FTLD is both pathologically and clinically heterogeneous [2-6]. Diseases in the FTLD spectrum involve cortical areas that have been implicated in olfactory processing in functional imaging studies of healthy subjects [7-14] and in human lesion studies [15-18]. Deficits of olfactory processing have been described in a number of common neurodegenerative conditions, including idiopathic Parkinson's disease and Alzheimer's disease [7,8]. Besides contributing to our understanding of the organisation of human olfactory processing, such deficits are of diagnostic value and have implications for clinical management [8]. However, very little information is available concerning olfactory processing in FTLD [19]. This information could potentially assist both in clinical diagnosis (often difficult in FTLD) and in understanding the pathogenesis of behavioural and affective symptoms [5]. Here we describe a detailed study of olfactory processing in patients clinically diagnosed with FTLD.

Correspondence to: Dr. Jason Warren Dementia Research Centre Institute of Neurology London WC1N 3BG United Kingdom Tel: 44 2078298773 Fax: 442076762066 Email: jwarren@dementia.ion.ucl.ac.uk. 


\section{Subjects and methods}

\section{Subjects}

Three right-handed British male patients (mean age 70 years; range 66 - 72 years) meeting consensus criteria for FTLD [20] and four healthy right-handed British male control subjects (mean age 69.5 years; range 69 - 70 years) participated. All participants gave signed informed consent and the study was approved by the local institutional Joint Research Ethics Committee. None of the participants was taking drugs likely to interfere with olfactory function, none was a smoker, and none had a history of other medical conditions known to be associated with olfactory dysfunction. Clinical information and brain magnetic resonance imaging (MRI) findings for the FTLD patients are summarised in Table 1. Patient 1 had a progressive behavioural syndrome and disproportionate bilateral anterior temporal lobe atrophy on MRI; Patient 2 had progressive breakdown in language comprehension (semantic dementia) with predominantly left-sided antero lateral temporal lobe atrophy on MRI; and Patient 3 had progressive impairment of speech production (progressive non-fluent aphasia) with accentuated left peri-Sylvian atrophy on MRI.

\section{General neuropsychometry}

All patients underwent general neuropsychometry (Table 1). Disease severity was assessed using the Folstein Mini-Mental State Examination (MMSE) Test [21]; verbal and nonverbal memory were assessed using the Recognition Memory Test (RMT) for words and faces [22] respectively; face identification (an index of semantic processing in another sensory domain, namely vision) was assessed using the Famous Faces test, which requires recognition and naming of photographs of 12 national and international public figures [EK Warrington, personal communication]; and word retrieval (an index of verbal capacity) was assessed using the Graded Naming Test (GNT) [23].

\section{Olfactory symptom questionnaire}

All patients and controls completed a structured questionnaire concerning olfactory function. Participants were asked to indicate whether they had noticed any change in their sense of smell, whether odours seemed less intense, strange, unpleasant or otherwise altered, whether things tended to smell the same, whether there had been any situations in which they had failed to detect odours detected by others, and when the symptoms began. Patients were assisted in completing the questionnaire by their accompanying spouse. Only Patient 1 described olfactory symptoms: this patient reported decreased odour intensity and reduced ability to detect odours, and complained that 'things smell the same'; these symptoms began around the same time as his other cognitive and behavioural symptoms.

\section{Odour identification}

Odour identification was assessed in patients and controls using the University of Pennsylvania Smell Identification Test (UPSIT) $[24,25]$. The UPSIT is a widely used olfactory assessment battery that has been validated in healthy populations and in many disease states [7], and age and gender norms are available [25]. The standard version of the test employs 40 common odorants embedded in 'scratch and sniff' fragrance labels and administered in a forced choice format in which the subject must select the name of the target odour from four alternatives. Cultural factors influence performance on all tests of olfactory identification including the UPSIT [26], which was designed for the North American population: in this study, we provided alternative, more familiar descriptions of a small number of items that were predicted to be unfamiliar to British people in the age cohort (for example, 'ginger ale' was substituted for 'root beer'). All subjects were administered the odours from the UPSIT booklets using the recommended examiner-assisted method of bilateral odour presentation [25]. 
We designed two modifications of the standard published version of the UPSIT to address specific issues relevant to the FTLD group that could potentially affect the interpretation of the findings. Patients with FTLD often have specific language difficulties: we therefore created a parallel visual version of the UPSIT, as an alternative measure of odour identification in a nonverbal domain. In the visual version of the UPSIT, subjects were required to select the picture corresponding to the target odour from four alternative picture stimuli. In addition, because both the verbal and the visual versions of the UPSIT require responses based on crossmodal matching, we tested subjects' capacity to perform a cross-modal matching task in modalities other than olfaction, in order to assess the specificity of any olfactory deficit. We created a picture-word matching test derived from the UPSIT, in which the subject was required to select the name of a target picture (corresponding to an UPSIT odour) from four alternative written words. The following fixed order of tests was used for all subjects: word version of the UPSIT, picture version of the UPSIT, picture-word matching test. No feedback was given to subjects about their performance on any trial.

\section{Odour discrimination}

Deficits in processing sensory information in FTLD could in principle arise at perceptual or semantic levels of analysis [8,19]: a deficit of odour identification detected on the UPSIT might therefore be due to defective perceptual encoding of olfactory information, or at a later processing stage at which that information is associated with meaning and identification is achieved. To distinguish perceptual from semantic levels of olfactory processing, we designed a perceptual test of odour discrimination that did not require explicit odour identification, based on the target odours used in the UPSIT and sampling from different odour classes [24]. Subjects were presented by the examiner on consecutive trials with 11 odours from the UPSIT booklet (banana, cherry, coconut, fruit punch, leather, menthol, mint, motor oil, orange, pizza, strawberry). The task on each trial was to decide whether the odour was the same or different from the preceding trial. The order of odours and of 'same'- 'different' odour presentations was randomised. In order to establish that reliable discrimination could be achieved via at least one nostril, odours were presented unilaterally to each nostril while the subject's other nostril was occluded by Micropore ${ }^{\mathrm{TM}}$ tape; 20 trials ( 10 'same', 10 'different') using the same set of odours were administered to each nostril, the side of presentation was alternated after every 10 trials, and the inter-trial interval was approximately five seconds.

\section{Results}

\section{General neuropsychometry}

Results of general neuropsychometry in the patient group are summarised in Table 1. Impaired performance is here considered conventionally as performance less than the $5^{\text {th }}$ percentile for age-matched healthy control subjects. On the MMSE and GNT, which depend on the integrity of verbal processing, Patient 1 performed comparably to healthy controls, whereas Patients 2 and 3 showed deficits attributable to impaired language processing. On the RMT for words and faces and the famous face identification test all patients performed normally $\left(>10^{\text {th }}\right.$ percentile for age).

\section{Odour identification}

Results of the tests of olfactory processing for both the patient and control groups are presented in Table 2. On the standard (word) version of the UPSIT, Patient 3 performed normally $\left(>10^{\text {th }}\right.$ percentile based on published age and gender norms [25]), while Patients 1 and 2 showed impaired performance. Healthy controls all performed normally on this test.

While published norms are not available for the visual version of the UPSIT, this test is intrinsically more difficult than the word version since a number of the UPSIT items are low- 
frequency items in the visual modality. This relative difficulty is reflected in the uniformly inferior performance of the healthy control sample on the visual version of the UPSIT (range 22-28/40) compared with the word version of the test (Table 2). In the FTLD group, Patients 2 and 3 showed impaired performance (Patient 1 declined to complete this test). These results suggest that impaired odour identification in FTLD is not attributable to a defect of verbal labelling. All patents performed comparably to healthy controls on the picture-word crossmodal matching test, indicating that defective odour identification in FTLD is not simply a consequence of impaired cross-modal matching, but rather reflects a modality-specific deficit of olfactory processing.

\section{Odour discrimination}

On the perceptual test of odour discrimination, patients performed comparably to controls: all achieved at least $75 \%$ correct performance via one nostril. This suggests that the deficit of odour identification in FTLD has a semantic rather than a perceptual basis (i.e., it is likely to be a disorder of olfactory knowledge rather than sensory processing).

\section{Discussion}

This study presents evidence for central deficits of odour identification in patients with FTLD. Our findings suggest that impaired olfactory function is particularly associated with temporal lobe involvement in FTLD. The findings further suggest that the odour identification deficit is a specific impairment of olfactory knowledge rather than being attributable to defective olfactory perception, or a more generalised impairment of cross-modal matching or verbal labelling. While patient numbers in our study were small, odour deficits do not appear to be strongly correlated with other types of nonverbal knowledge (for example, face identification: Tables 1 and 2). Our findings await corroboration in larger prospective cohorts, however the present evidence supports current models of the organisation of human olfactory cortex emerging from functional imaging studies [7-14] which have implicated a bilateral network of areas including the antero-mesial temporal lobes. These regions are sites of heavy disease involvement in FTLD [2].

The present findings suggest that olfactory impairment is more severe (and becomes more clinically salient) with increasing involvement of the temporal lobes. Patient 1 , who had a severe impairment of odour identification and was also the only member of the FTLD group who reported olfactory symptoms, had bilateral temporal lobe atrophy on MRI. Patient 2 had asymmetric (predominantly left-sided) temporal lobe atrophy and also showed deficits on both the word and visual versions of the UPSIT. In contrast Patient 3, with asymmetric atrophy predominantly involving the peri-Sylvian region (superior temporal and inferior frontal lobes) performed normally on the word version of the UPSIT, though he was impaired on the more difficult visual version. This evidence suggests that conceptual knowledge about odours may have a brain organisation analogous to other types of nonverbal knowledge (for example, faces), based on a distributed brain network within which particular regions might be relatively selective for odour processing [27]. Our study does not allow clear conclusions regarding hemispheric lateralisation of olfactory processing. A preferential role for the right hemisphere in both the perceptual and semantic analysis of odours is supported by functional imaging evidence in normal subjects [9,12], and psychophysical evidence in normal subjects [28] and in patients with focal cerebral resections [15,17]. However, other evidence $[7,10,11,13,18]$ argues against a simple serial transfer of olfactory information within or between hemispheres.

Our findings have important clinical implications. Only one of the FTLD patients in this sample who exhibited a psychophysical defect of olfactory processing had symptoms suggestive of an olfactory disorder. Central olfactory abnormalities may be more common than previously suspected in patients with FTLD, and may elude detection at the bedside, particularly if the 
patient is unaware of or cannot describe the defect (for example, Patient 2 here). Detection of such deficits could facilitate early diagnosis in this diverse clinical population, in which prominent behavioural abnormalities often suggest a primary psychiatric rather than neurodegenerative process. Distorted processing of olfactory signals might contribute to the abnormal emotional responses that are a striking feature in many patients with FTLD, in particular the temporal lobe variant [5]. Moreover, unrecognised olfactory deficits have potentially serious safety and nutritional implications. Our findings suggest that a more detailed prospective assessment of olfactory function is justified in patients with FTLD, and this is likely to be particularly relevant to individuals with disease predominantly involving the temporal lobes.

\section{Acknowledgments}

We are grateful to the patients and their carers for their participation. This study was supported by the Alzheimer Research Trust and by the Frognal Trust, London. JDW is supported by EU contract LSHM-CT-2003-503330 (The APOPIS Consortium). The authors thank Dr. Jane Warren and Prof. Elizabeth Warrington for helpful discussion, Prof. Martin Rossor for permission to study patients under his care, and Ms. Shona Price for assistance in preparation of the brain images.

\section{References}

1. Ratnavalli E, Brayne C, Dawson K, Hodges JR. The prevalence of frontotemporal dementia. Neurology 2002;58:1615-1621. [PubMed: 12058088]

2. Whitwell JL, Josephs KA, Rossor MN, Stevens JM, Revesz T, Holton JL, Al-Sarraj S, Godbolt AK, Fox NC, Warren JD. Magnetic resonance imaging signatures of tissue pathology in frontotemporal dementia. Arch Neurol 2005;62:1402-1408. [PubMed: 16157747]

3. Hodges JR, Davies R, Xuereb J, et al. Clinicopathological correlates in frontotemporal dementia. Ann Neurol 2004;56:399-406. [PubMed: 15349867]

4. Rosen HJ, Gorno-Tempini ML, Goldman WP, et al. Patterns of brain atrophy in frontotemporal dementia and semantic dementia. Neurology 2002;58:198-208. [PubMed: 11805245]

5. Rosen HJ, Perry RJ, Murphy J, et al. Emotion comprehension in the temporal variant of frontotemporal dementia. Brain 2002;125:2286-2295. [PubMed: 12244085]

6. Gorno-Tempini ML, Dronkers NF, Rankin KP, et al. Cognition and anatomy in three variants of primary progressive aphasia. Ann Neurol 2004;55:335-346. [PubMed: 14991811]

7. Doty RL. Olfaction. Annu Rev Psychol 2001;52:423-452. [PubMed: 11148312]

8. Hawkes C. Olfaction in neurodegenerative disorder. Mov Disord 2003;18:364-372. [PubMed: 12671941]

9. Zatorre RJ, Jones-Gotman M, Evans AC, Meyer E. Functional localization and lateralization of human olfactory cortex. Nature 1992;360:339-340. [PubMed: 1448149]

10. Royet JP, Koening O, Gregoire MC, et al. Functional anatomy of perceptual and semantic processing for odours. J Cogn Neurosci 1999;11:94-109. [PubMed: 9950717]

11. Gottfried JA, Deichmann R, Winston JS, Dolan RJ. Functional heterogeneity in human olfactory cortex: an event-related functional magnetic resonance imaging study. J Neurosci 2002;22:1081910828. [PubMed: 12486175]

12. Savic I. Brain imaging studies of the functional organization of human olfaction. Neuroscientist 2002;8:204-211. [PubMed: 12061500]

13. Kareken DA, Mosnik DM, Doty RL, Dzemidzic M, Hutchins GD. Functional anatomy of human odour sensation, discrimination, and identification in health and aging. Neuropsychology 2003;17:482-495. [PubMed: 12959514]

14. Zelano C, Sobel N. Humans as an animal model for systems-level organization of olfaction. Neuron 2005;48:431-454. [PubMed: 16269361]

15. Zatorre RJ, Jones-Gotman M. Human olfactory discrimination after unilateral frontal or temporal lobectomy. Brain 1991;114:71-84. [PubMed: 1998891] 
16. Jones-Gotman M, Zatorre RJ. Odour recognition memory in humans: role of right temporal and orbitofrontal regions. Brain Cogn 1993;22:182-198. [PubMed: 8373572]

17. West SE, Doty RL. Influence of epilepsy and temporal lobe resection on olfactory function. Epilepsia 1995;36:531-542. [PubMed: 7555965]

18. Jones-Gotman M, Zatorre RJ, Cendes F, et al. Contribution of medial versus lateral temporal-lobe structures to human odour identification. Brain 1997;120:1845-1856. [PubMed: 9365374]

19. Mendez MF, Ghajarnia M. Agnosia for familiar faces and odours in a patient with right temporal lobe dysfunction. Neurology 2001;57:519-521. [PubMed: 11502924]

20. McKhann GM, Albert MS, Grossman M, Miller B, Dickson D, Trojanowski JQ. Clinical and pathological diagnosis of frontotemporal dementia: report of the Work Group on Frontotemporal Dementia and Pick's Disease. Arch Neurol 2001;58:1803-1809. [PubMed: 11708987]

21. Folstein MF, Folstein SE, McHugh PR. "Mini-mental state": a practical method for grading the cognitive state of patients for the clinician. J Psychiatr Res 1975;12:189-198. [PubMed: 1202204]

22. Warrington, EK. Recognition Memory Test. NFER-Nelson; Windsor (UK): 1984.

23. Warrington EK. The Graded Naming Test: A restandardisation. Neuropsychol Rehab 1997;7:143146.

24. Doty RL, Shaman P, Dann M. Development of the University of Pennsylvania Smell Identification Test: a standardized microencapsulated test of olfactory function. Physiol Behav 1984;32:489-502. [PubMed: 6463130]

25. Doty, RL. The Smell Identification Test ${ }^{\mathrm{TM}}$ Administration Manual. 3rd Edition. Sensonics, Inc.; Haddon Hts (NJ): 1995.

26. Doty RL, Marcus A, Lee WW. Development of the 12-item Cross-Cultural Smell Identification Test (CC-SIT). Laryngoscope 1996;106:353-356. [PubMed: 8614203]

27. Snowden JS, Thompson JC, Neary D. Knowledge of famous faces and names in semantic dementia. Brain 2004;127:860-872. [PubMed: 14985259]

28. Zatorre RJ, Jones-Gotman M. Right-nostril advantage for discrimination of odours. Percept Psychophys 1990;47:526-531. [PubMed: 2367173] 

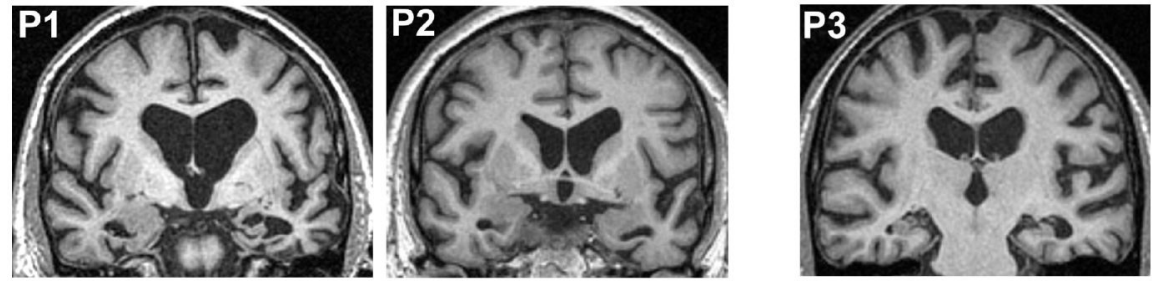

Figure 1.

Brain magnetic resonance imaging findings in FTLD patients. All images are T1-weighted coronal sections, presented in radiological convention (the left hemisphere is on the right). Patients 1 and 2 (P1 and P2) have temporal variant FTLD clinically and radiologically, while Patient 3 (P3) has progressive non-fluent aphasia. P1: disproportionate bilateral temporal lobe atrophy on a background of more diffuse atrophy. P2: disproportionate asymmetric atrophy of left anterior temporal lobe. P3: accentuated left peri - Sylvian atrophy. 


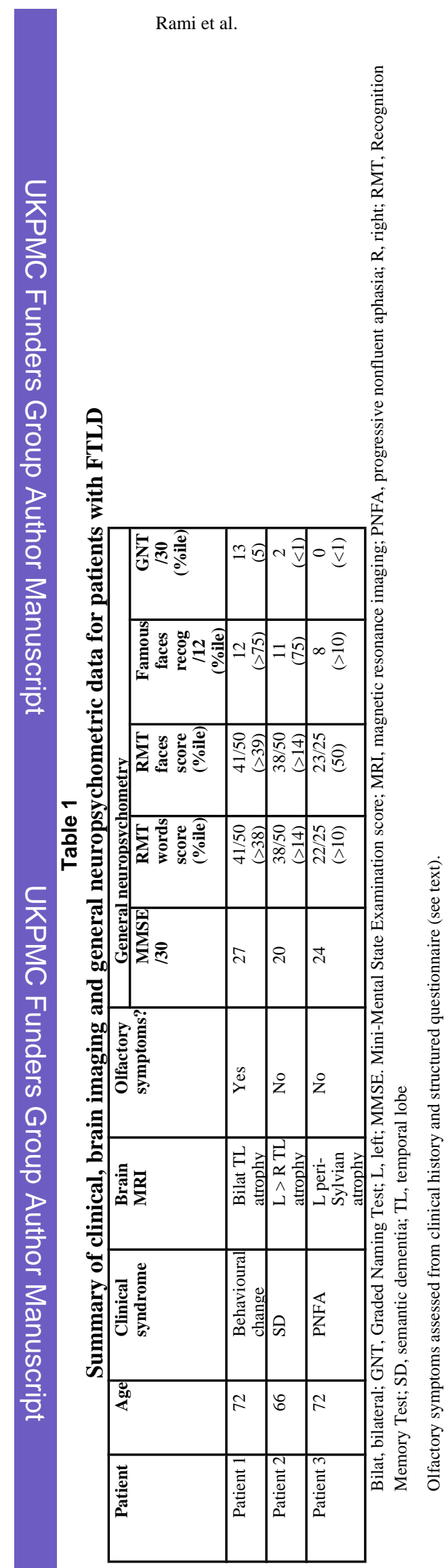

Page 8

$J$ Neurol. Author manuscript; available in PMC 2008 October 14. 


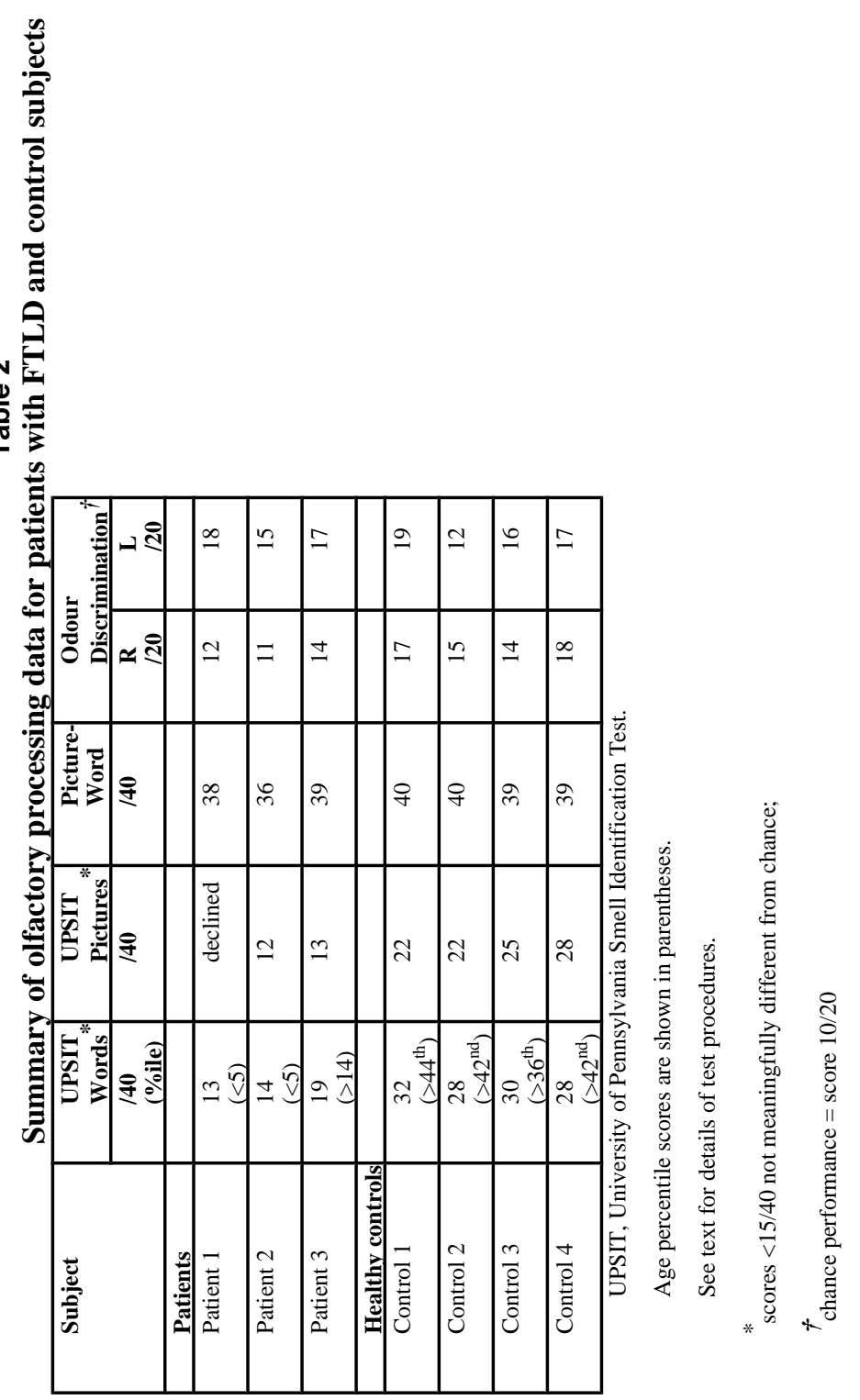

